\title{
PRIMARY ELEMENTS AND PRIME POWER ELEMENTS IN MULTIPLICATIVE LATTICES
}

\author{
C. JAYARAM AND E. W. JOHNSON
}

Throughout, we assume that $L$ is a $C$-lattice. For any prime element $p$ of $L, L_{p}$ denotes the localization at $\{x \in C \mid x \leq x p$, where $C$ is a multiplicative closed subset of compact elements of $L$ which generates $L$ under joint. For details see [7].

We shall begin with the following definitions.

Definition 1. $L$ is said to satisfy the conditon $(\alpha)$ if every primary element is a power of its radical.

Definition 2. $L$ is said to satisfy the condition $(\delta)$ if every element is a finite meet of prime power elements.

Let $R$ be a commutative ring with identity. If every primary ideal of $R$ is a power of its radical, then $L(R)$ (the lattice of ideals of $R$ ) is an r-lattice satisfying the conditon $(\alpha)$. If every ideal of $R$ is a finite intersection of prime power ideals, then $L(R)$ is an r-lattice whcih satisfies the condition $(\delta)$. If $L$ is a principally generated $M$-lattice (for definition see [6]), then $L$ satisfies the condition $(\alpha)$ (see Lemma 4.4 of [2]). If $L$ is a principal element lattice, then $L$ satisfies the condition $(\delta)$ (see Theorem 5 of [8]).

Craig A. Wood, H. S. Butts and R. W. Gilmer have studied these conditions in the case of commutative rings (see [3] and [4]).

We need some more definitions to prove the main results.

Defintion 3. A prime element $p$ of $L$ is said to be an $\alpha$-prime if every primary element $q \leq p$, is a power of its radical.

Definition 4. A prime element $p$ of $L$ is called a weak $\delta$-prime if every element $a \leq p$ is a finite meet of prime power elements.

Definition 5. A prime element $p$ of $L$ is called a $\delta$-prime if every element $a \leq p$ is a finite meet of powers of $\alpha$-prime elements.

Received September 20, 1994; revised October 3, 1995. 
Note that $L$ satisfies the condition $(\alpha)$ if and only if every prime is an $\alpha$-prime. Obviously, every $\delta$-prime element is a weak $\delta$-prime element.

We prove some lemmas that we need.

Lemma 1. If $p$ is a weak $\delta$-prime element, then $p$ is an $\alpha$-prime element.

Proof. The proof of the lemma is similar to the proof of Theorem 8 of [3].

Lemma 2. The following statements on $L$ are equivalent:

(i) $L$ satisfies the conditon $(\delta)$.

(ii) Every prime element of $L$ is a weak $\delta$-prime element.

(iii) Every prime element is a $\delta$-prime element

Proof. (i) $\Leftrightarrow$ (ii) directly follows from the defintions and (iii) $\Rightarrow$ (ii) is obvious. (ii) $\Rightarrow$ (iii) follows from Lemma 1.

If $\left\{p_{\alpha}\right\}$ is the collection of prime elements minimal over $a$, then by the isolated primary component of $a$ belonging to $\dot{p}_{\alpha}$ (or the isolated $p_{\alpha}$-primary component of $a$ ) we mean the meet $\wedge q_{\alpha}$ of all $p_{\alpha}$-primary elements which contain $a$.

Note that in $L$, every finite product of compact elements is compact. Therefore if $a \leq p$ and $p$ is a prime element, then $p$ is a minimal prime over $a$ if and only if for any compact element $x \in L, x \leq p$ implies there exists a compact element $y \not z p$ such that $x^{n} y \leq a$ for some positive integer $n$ (see Lemma 3.5 of [2]). Further if $p$ is a minimal prime over $a$, then the isolated $p$-primary component of $a$ is a $p$-primary element (see Lemma 3.8 of [2]).

Lemma 3. Let $p$ be a minimal prime over $a(a, p \in L)$ and let $q$ be the isolated p-primary component of a. Then

$$
q=\bigvee\{x \in C \mid x y \leq a, y \leq p \quad \text { for some } y \in C\}=a_{p}
$$

Proof. The proof of the lemma is straightforward and hence is omitted.

Lemma 4. Let $p$ be a prime element of L. For any $n \in \mathbb{Z}^{+}$. let $p^{(n)}=\bigvee\{x \in$ $C \mid x y \leq p^{n}, y \leq p$ for some $\left.y \in C\right\}$. Then $p^{(n)}$ is the isolated primary component of $p^{n}$.

Proof. The proof of the lemma follows from Lemma 3.

For each $n \in \mathbb{Z}^{+}, p^{(n)}$ is called the $n$th symbolic power of $p$. Note that $p^{(n)}=p_{p}^{n}$ and $p^{(n)} \circ_{p} p^{(k)}=p_{p}^{n} \circ p_{p}^{k}=\left(p^{n} p^{k}\right)_{p}=p_{p}^{n+k}=p^{(n+k)}$.

Lemma 5. Let $p$ be a prime element of $L$ and let every p-primary element be a power of $p$. If the symbolic powers of p properly descend, then for each $n \in \mathbb{Z}^{+}, p^{(n)}=p^{n}$. Hence each $p^{n}$ is p-primary. 
Proof. The proof follows by induction on $n$.

For each prime element $p \in L$, Let $p^{w}=\bigwedge_{n=1}^{\infty} p^{n}$ and $p^{(w)}=\bigwedge_{n=1}^{\infty} p^{(n)}$.

Lemma 6. Let $p$ be a prime element of $L$. If $p^{(w)}$ is a prime element and if $p^{(w)}<p$, then the symbolic powers of $p$ properly descend.

Proof. Observe that $p^{(w)}=\bigwedge_{n=1}^{\infty} p^{(n)}=\bigwedge_{n=1}^{\infty} p_{p}^{n}$, so $p^{(w)}=p_{p}^{(w)}=\left(\bigwedge_{n=1}^{\infty} p_{p}^{n}\right)_{p}=$ $\bigwedge_{p} p_{p}^{n}=\bigwedge_{p} p^{(n)}$. If $p^{(n)}=p^{(n+1)}=p^{(n)} \circ_{p} p^{(1)}$, then $p^{(n)}=p^{(k)}$ for all $k \geq n$, so $p^{(w)}=p^{(n)}$. Since $p^{n} \leq p^{(n)}=p^{(w)}$ and $p^{(w)}$ is a prime element, it follows that $p \leq p^{(w)}$ which is a contradiction. Therefore the symbolic powers of $p$ properly descend. This completes the proof the lemma.

An element $a \in L$ is said to be a strong join principal element if $a$ is join principal and compact. An element $b \in L$ is called prime to $a(a<1)$ if whenever $b c \leq a$, then $c \leq a$.

Lemma 7. Let $d$ be a strong join principal element of $L$ and let $d$ be prime to an element $b \in L$. Suppose $p$ is a prime element minimal over $d \vee b$ and let $q_{i}$ be the isolated p-primary component of $d^{i} \vee b$. Then $q_{1}>q_{2}>q_{3}>\cdots$.

Proof. Clearly $q_{1} \geq q_{2} \geq \cdots$. We show that for each $i, d^{i} \leq q_{i}$ and $d^{i} \not q_{i+1}$. Obviously $d^{i} \leq q_{i}$. If $d^{i} \leq q_{i+1}$, then by Lemma $3, d^{i} y \leq d^{i+1} \vee b=d^{i} d \vee b$ for some compact element $y \notin p$. Since $d^{i}$ is prime to $b$, it follows that $\left(b: d^{i}\right) \leq b$. As $y \leq\left(d^{i} d \vee b: d^{i}\right)$ and $d^{i}$ is join principal, we get $y \leq d \vee\left(b: d^{i}\right) \leq d \vee b \leq p$ which is a contradiction. Therefore $d^{i} \leq q_{i+1}$ and hence $q_{1}>q_{2}>q_{3}>\cdots$.

Theorem 1. Let $p$ be a prime element of $L$ and let every p-primary element be a power of its radical. Let $d$ be a strong join principal element and let $d$ be prime to an element $b \in L$. If $p$ is minimal prime over $d \vee b$, then the powers of $p$, properly descend, $b \leq p^{w}$ and $p^{w}$ is the meet of all p-primary elements of $L$.

Proof. The proof of the theorem is simsilar to the proof of Theorem 1 of [3].

Theorem 2. Suppose $L$ is generated by strong join principal elements. Let $m$ be an $\alpha$-prime element and let $p_{0}$ be a prime element such that $p_{0}<m$. Then $p_{0} \leq m^{w}$.

Proof. Choose any strong join principal element $d$ such that $d \leq m$ and $d \leq p_{0}$. Then $d$ is prime to $p_{0}$. Let $p \leq m$ be a minimal prime over $d \vee p_{0}$. Then by Theorem 1 , $p_{0} \leq p^{w} \leq m^{w}$. This completes the proof of the theorem.

Lemma 8. Suppose $L$ is generated by strong join principal elements. Let $p$ and $m$ be prime elements such that $p<m$ and there are no prime elements strictly between $p$ and $m$. If every m-primary element is a power of $m$, then 
$p=\bigwedge_{k=1}^{\infty} m^{(k)}=\bigwedge_{k=1}^{\infty} m^{k}$.

Proof. We show that $\bigwedge_{k=1}^{\infty} m^{(k)} \leq p$. Let $x$ be any strong join principal element such that $x \leq \bigwedge_{k=1}^{\infty} m^{(k)}$ and $x \not p$. Then $x^{2} \not \leq p$, so $m$ is a minimal prime over $p \vee x^{2}$. Let $q=\bigvee\left\{y \in C \mid y z \leq x^{2} \vee p, z \notin m\right.$, for some $\left.z \in C\right\}$. The $q$ is $m$-primary, so by hypothesis, $q=m^{k}$ for some $k \in \mathbb{Z}^{+}$. As $m^{k}$ is $m$-primary, it follows that $m^{k}=m^{(k)}$ and hence $x \leq q$. As $x$ is compact, $x a \leq x^{2} \vee p$ for some $a \leq m$. As $x$ is join principal, $a \leq\left(x^{2} \vee p: x\right)=x \vee(p: x) \leq x \vee p$ since $(p: x) \leq p$. Therefore $a \leq p \vee x \leq m$, a contradiction and hence $\bigwedge_{k=1}^{\infty} m^{(k)} \leq p$. The remaining part follows from Theorem 1 .

Theorem 3. Let $L$ be generated by strong join principal elements. Let $m$ be a nonminimal prime element. If $m$ is an a-prime, then $m^{w}=\bigwedge_{k=1}^{\infty} m^{k}$ is a prime element containing each prime element properly contained in $m$. Further each $m^{k}$ is primary.

Proof. If $m=m^{2}$, the we are through. So assume that $m^{2}<m$. Let $\mathcal{Y}=\{p \in L \mid$ $p$ is prime and $p<m\}$. By hypothesis $\mathcal{Y} \neq \emptyset$. By Theorem 2 and by Zorn's lemma, $\mathcal{Y}$ contains a maximal element $p$ such that $p$ is prime, $p<m$ and there are no prime elements properly between $p$ and $m$. By Lemma $8, p=m^{w}=m^{(w)}$. By Theorem $2, p$ contains each prime element properly contained in $m$. Further by Lemma 5 and Lemma 6 , each $m^{k}$ is $m$-primary. This completes the proof of the theorem.

As consequences, we have the following results.

Corollary 1. Let $L$ be generated by strong join principal elements. If $L$ satisfies the condition $(\alpha)$, then $p^{n}\left(n \in \mathbb{Z}^{+}\right)$is p-primary for every nonminimal prime element $p$ of $L$.

Corollary 2. Let $L$ be generated by strong join principal elements. If $L$ is a domain and if $p$ is an $\alpha$-prime, then $p^{n}$ is $p$-primary for each $n \in \mathbb{Z}^{+}$.

Corollary 3. Let $L$ be generated by strong join principal elements. If $L$ is a domain and if $L$ satisfied the condition $(\alpha)$, then prime power elements are primary.

Lemma 9. Suppose $L$ is generated by strong join principal elements. Let $\sqrt{a}=p$ and $p$ be a $\delta$-prime element. Then $a=p^{n}$ for some $n \in \mathbb{Z}^{+}$.

Proof. By using Theorem 2 and by imitating the proof of Theorem 9 of [3], we can get the result.

Lemma 10. Suppose $L$ is generated by strong join principal elements. If $p$ is a nonminimal $\delta$-prime element, then $p$ is maximal.

Proof. Suppose $p$ is a nonminimal $\delta$-prime element. Then $p_{1}<p$ for some prime 
element $p_{1} \leq L$. Choose any strong join principal element $d \leq p$ such that $d \not \leq p$. Let $p_{0} \leq p$ be a minimal prime over $d \vee p_{1}$. Note that $p_{0}$ is a $\delta$-prime and hence it is an $\alpha$-prime. Again by Lemma $7, p_{0} \neq p_{0}^{2}$. Choose any strong join principal $y \leq p_{0}$ such that $y \not z p_{0}^{2}$. Suppose $p$ is nonmaximal. Then $p<m$ for some maximal element $m$ of $L$. Since by Theorem $3, p_{0}^{2}$ is $p_{0}$-primary, $m y \leq p_{0}^{2}$, so $p_{0}^{2}<p_{0}^{2} \vee m y \leq p_{0}$ and therefore $\sqrt{p_{0}^{2} \vee m y}=p_{0}$. By Lemma $9, p_{0}^{2} \vee m y=p_{0}$, so $y \leq m y \vee p_{0}^{2}$ and hence $1=\left(m y \vee p_{0}^{2}: y\right)$. Again since $y$ is join principal, we have $1=m \vee\left(p_{0}^{2}: y\right) \leq m \vee p_{0}\left(\right.$ since $\left.\left(p_{0}^{2}: y\right) \leq p_{0}\right)=m$, a contradiction. Therefore $p$ is maximal and this completes the proof of the lemma.

For the definitons of discrete valuation lattices and special principa element lattices, the reader is referred to [10].

Lemma 11. Suppose $L$ is principally generated. If $m$ is a nonminimal $\delta$-prime element, then $L_{m}$ is a one-dimensional discrete valuation lattice.

Proof. Note that by Lemma $10, \operatorname{dim} L_{m}=1$, so every element has a prime radical. Again as $m$ is a $\delta$-prime, by Lemma 9, every element of $L_{m}$ is a prime power. Consequently $L_{m}$ is totally ordered. Again by Lemma 4.8 of [1], $L_{m}$ is a one-dimensional discrete valuation lattice.

Lemma 12. Suppose $L$ is principally generated. If $m$ is a $\delta$-prime element whcih is both maximal and minimal, then $L_{m}$ is a special principal element lattice.

Proof. The proof of the lemma follows from Lemma 9.

Theorem 4. Suppose $L$ is principally generated. Then the following statements are equivalent:

(i) L satisfies the condition $(\delta)$.

(ii) Every prime element is a weak $\delta$-prime element.

(iii) Every prime element is a $\delta$-prime element.

(iv) Every element is principal.

Proof. By Lemma 2, (i), (ii), and (iii) are equivalent, (iii) $\Rightarrow$ (iv). Suppose (iii) holds. By Lemma 10, $\operatorname{dim} L \leq 1$. Next we show that every prime element is weak meet principal. Let $m$ be a prime element. Suppose $a \leq m$. By hypothesis, $a=p_{1}^{\alpha_{1}} \wedge \cdots \wedge p_{n}^{\alpha_{n}}$ for some prime elements $p_{i} \in L(i=1,2, \ldots, n)$. Without loss of generality, assume that $p_{i}{ }^{\prime}$ s are distinct.

We can also assume that $p_{i} \leq p_{j}$ for $i \neq j(1 \leq i, j \leq n)$. By Lemma 1 , every prime element is an $\alpha$-prime. As $\operatorname{dim} L \leq 1$, by Theorem $3, p_{i}$ 's are comaximal and hence $a=p_{1}^{\alpha_{1}} \cdot p_{2}^{\alpha_{2}} \cdots p_{n}^{\alpha_{n}}$. As $a \leq m, p_{i} \leq m$ for some $i$. If $p_{i}=m$, then we are through. Suppose $p_{i}<m$. By Lemma $11, p_{i_{m}}=0_{m}$ and so $a b=0$ for some $b \leq m$. Since $m$ is maximal, it follows that $a=a m$. This shows that every prime element is weak meet principal. Again note that $L$ contains only a finite number of minimal primes and hence 
by Theorem 1.5 of [9], $L$ is a principal element lattice. (iv) $\Rightarrow$ (iii) follows from Theorem 5 of [8]. This completes the proof of the theorem.

\section{References}

[1] D. D. Anderson, "Abstract commutative ideal theorey without chain condition," Algebra Universalis, 6(1976), 131-145.

[2] D. D. Anderson, C. Jayaram and F. Alarcon, "Some results on abstract commutative ideal theory," Stu. Sci. Math. Hung. (to appear).

[3] H. S. Butts and R. W. Gilmer, "Primary ideals and prime power ideals," Canad. J. Math., (1966), 1183-1195.

[4] Craig A. Wood, "On general ZPI-rings," Pacific J. Math., 30(1969), 837-846.

[5] R. P. Dilworth, "Abstract commutative ideal theory," Pacific J. Math, 12(1962), 481-498.

[6] M. F. Janowitz, "Principal multiplicative lattices," Pacific J. Math., 33(1970), 653-656.

[7] C. Jayaram and E. W. Johnson, "Almost principal element lattices," Inter. J. Math. and Math. Sci. (to appear).

[8] E. W. Johnson and J. P. Lediaev, "Representable distributive Noether lattices," Pacific J. Math., 28(1969), 561-564.

[9] E. W. Johnson and J. A. Johnson, "P-lattices as ideal lattices and submodule lattices," Commen. Math. Univ. San. Pauli, 38(1989), 21-27.

[10] E. W. Johnson, "Selfduality and comultiplication lattices," Algeb̆ra Universalis, 26(1989), 196-201.

The University of Iowa, Department of Methematics, Iowa City, Iowa 52242, U.S.A. 